\title{
Response to: "Hysteroscopic metroplasty: reproductive outcome in relation to septum size" by Paradisi et al.
}

\author{
Matthew Prior • Nick Raine-Fenning
}

Received: 4 April 2014 / Accepted: 5 May 2014 / Published online: 20 May 2014

(C) Springer-Verlag Berlin Heidelberg 2014

To the Editor:

We recently read with interest the article by Paradisi et al. entitled "Hysteroscopic metroplasty: reproductive outcome in relation to septum size" [1]. This study aims to determine if the reproductive performance after hysteroscopic resection of partial uterine septum was related to septum size and concludes that removal of partial septa improves reproductive outcome irrespective of previous obstetric history.

A Cochrane review of metroplasty versus expectant management in women with recurrent miscarriage and a septate uterus found insufficient evidence to support this treatment as no controlled trials have been performed [2].

The design of the study used patients as their own controls. This approach is bias because the reason for performing resection is the poor reproductive history. These women could have miscarried by chance and still have a good prognosis without treatment.

The results state that all septa were "almost completely removed but some cases a septum remained". It is not clear whether this was assessed by hysteroscopy or 3D ultrasound and we would be interested to know how many patients had residual septa. Did subgroup analysis show that their reproductive outcome was significantly different to patients who had been deemed to have a full resection?

To build on this study a prospective randomised controlled trial of metroplasty versus conservative management with multiple surgeons would provide further evidence to advise women on the benefits of septum resection.

Conflict of interest None.

\section{References}

1. Paradisi R et al (2014) Hysteroscopic metroplasty: reproductive outcome in relation to septum size. Arch Gynecol Obstet 289(3):671-676

2. Kowalik CR et al (2011) Metroplasty versus expectant management for women with recurrent miscarriage and a septate uterus. Cochrane Database Syst Rev (6):CD008576

M. Prior $(\square)$

Department of Obstetrics and Gynaecology, Royal Preston Hospital, Lancashire Teaching Hospitals, Preston PR2 9HT, UK e-mail:mprior@me.com

\section{N. Raine-Fenning}

Nurture Fertility, Nottingham University Hospitals NHS Trust, Queen's Medical Centre Campus, Derby Road,

Nottingham NG7 2UH, UK 